\title{
Bootstrap Dependence on Plasma Profile Parameters
}

\author{
‥ Pomphrey \\ Princeton Plasma Phrsics Laboratory. Princeton University \\ Princeton. N. J. 08543
}

\begin{abstract}
The bootstrap fraction. $I_{b s} / I_{p}$. and the shape of the bootstrap current density. $J_{b s}(r)$, can depend sensitively on the shape of the plasma profiles. Beginning with Hirshman's form for the bootstrap current density, and making a large aspect ratio expansion (proceeding two orders in the square root of the inverse aspect ratio. $t$ ), we derive an expression for the bootstrap fraction of the form $I_{b s} / I_{p}=\epsilon^{1 / 2} 3_{p} C_{b s}\left(\alpha_{n}, \alpha_{T}: \alpha_{J} . Z, \epsilon\right)$ which depends explicitly on the peakedness of the density, temperature. and current. Examination of contours of constant $I_{b s} / I_{p}$ in the space of $\alpha_{n}, \alpha_{T}$ and consideration of the shape of the bootstrap current density help identify operating regimes for tokamaks which optimize bootstrap current drive.
\end{abstract}




\section{Introduction}

The possibility of using bootstrap current to decrease the external current drive requirements for tokamak reactors was first discussed by Bickerton. Connor, and Taylor ${ }^{1}$ in 1971 . Yet definitive evidence for substantial production of this current drive in tokamaks was not obtained until 1988 when its existence was confirmed in TFTR, ${ }^{2}$ and shortly thereafter on other major tokamaks. ${ }^{3-5}$

The improved ability for tokamaks to explore plasma regimes with augmented bootstrap current drive has motivated a number of recent publications which calculate the size of the bootstrap current in tokamaks. ${ }^{7-12}$ The present paper is a contribution which makes explicit the dependence of the bootstrap fraction $I_{h_{s}} / I_{p}$ on parameters $\alpha_{n}, \alpha_{T}$, and $\alpha_{J}$ describing the peakedness of the density, temperature, and equilibrium current profiles. In Sec. II. we derive an expression for $C_{b s}\left(\alpha_{n}, \alpha_{T} ; \alpha_{J}, \epsilon, Z\right) \equiv\left(I_{b s} / I_{p}\right) /\left(\epsilon^{1 / 2} \beta_{p}\right)$, a coefficient which relates the bootstrap current fraction to poloidal beta. $\beta_{p}$, at a given inverse aspect ratio, $\epsilon$, effective charge, $Z$. and equilibrium profile parameters $\alpha_{n}$. $\alpha_{T}$, and $\alpha_{J}$. To obtain $C_{b s}$ we perform a large aspect ratio expansion of Hirshman's collisionless form for the bootstrap current density ${ }^{17}$ and proceed through two orders in $\vee \epsilon$. Similar analyses were presented by DeVoto et al. ${ }^{8}$ and Pustovitov, ${ }^{9}$ who retained only the first order terms, and showed tabulated values of $C_{b s}$ for selected profile parameters. However the first order results can be shown to be inaccurate, especially when the density profile is flat so that $\alpha_{p} \approx \alpha_{T}$. Contour plots of $C_{b s}$ as a function of $n_{n}$ and $\alpha_{T}$ for fixed $\epsilon, Z$. and various $\alpha_{J}$ are presented in Fig. 1 and provide a comprehensive survey of this bootstrap coefficient.

It is desirable to operate tokamak reactors at high plasma beta, $\beta$, as well as with a high bootstrap current fraction, $I_{b s} / I_{p}$. However, since the poloidal beta, $\beta_{p}$, at the Troyon limit is inversely proportional to $\beta$, and since the bootstrap fraction is proportional to $\beta_{p}$, the desires for high $\beta$ and high $I_{b s} / I_{p}$ compete with one another ${ }^{13,14}$ It is natural, therefore, to consider the dependence of the bootstrap fraction $I_{b s} / I_{p}$ on profile parameters at constant beta. To do this, we absorb the current profile dependence of $\beta_{p}$ into the coefficient $C_{b s}$, express $\beta$ as a fraction of the Troyon limit, and contour the resulting expression for $I_{b s} / I_{p}$ at a fixed fraction of the beta limit (see Fig. 2). 
The benefits of achieving high values of $I_{b s} / I_{p}$ cannot be realized unless the shape of the bootstrap current profile is close to some desirable equilibrium current pronile. Since the bootstrap current density, $J_{b s}$, vanishes at the magnetic axis, a positive seed current must be supplied near the plasma axis by an external current drive scheme if a stable equilibrium current profile is to be obtained. In addition, the vanishing of the bootstrap current at the plasma edge implies the existence of a maximum of $J_{b s}$ at some intermediate radial location. It follows that if $I_{b s} / I_{p}$ is close to unity, then, unless the turning point location for $J_{b s}$ is near the magnetic axis, bootstrap "overdrive" in the region of the turning point is unavoidable (see Fig. 3), requiring the external current drive scheme to drive negative current near the bootstrap maximum. Clearly, "desirable" bootstrap current profiles which minimize the external current drive requirements will generally have maxima close to the magnetic axis. In Sec. III. we consider this alignment problem, and derive the dependence $x_{t}\left(\alpha_{n}, \alpha_{T}\right)$ of the location of the bootstrap turning point on profile parameters, so that contours of $x_{t}$ can be superimposed on the contours of constant $I_{b s} / I_{p}$. Thus, in a single contour plot, such as Fig. 2. we obtain information on both the magnitude and shape of the bootstrap current profile.

Finally, we remark that Boozer ${ }^{15,16}$ has recently argued that poloidal flux production by tearing mode activity near the center of a bootstrap driven tokamak with $I_{b s} / I_{p} \approx 1$ may be sufficient to sustain the plasma current without the need for an externally driven seed current. Efficient dynamo action would also require a bootstrap profile, $J_{b s}(r)$, which peaks close to the magnetic axis.

\section{The Bootstrap Fraction $I_{b s} / I_{p}$}

Our starting point is Hirshman's expression ${ }^{17}$ for the flux surface averaged parallel bootstrap current density, which is valid for zero collisionality, and arbitrary aspect ratio:

$$
\begin{aligned}
\frac{\langle\mathbf{J} \cdot \mathbf{B}\rangle_{b s}}{R\langle\mathbf{B} \cdot \nabla \phi\rangle} & =-\frac{R p_{e}(\psi)}{D}\left\{A_{1}\left[\frac{1}{p_{e}} \frac{d p_{e}}{d \psi}+\frac{1}{2} \frac{T_{i}}{T_{e}}\left(\frac{1}{p_{i}} \frac{d p_{i}}{d \psi}+\alpha_{i} \frac{1}{T_{i}} \frac{d T_{i}}{d \psi}\right)\right]\right. \\
& \left.-A_{2} \frac{1}{T_{e}} \frac{d T_{e}}{d \psi_{i}}\right\}
\end{aligned}
$$




$$
\begin{aligned}
f & =\frac{f_{t}}{\left(1-f_{t}\right)}, \\
\alpha_{i} & =-\frac{1.172}{(1.0+0.462 f)}, \\
A_{1} & =f\left(0.754+2.21 Z+Z^{2}\right)+f^{2}\left(0.348+1.2 .7 Z+Z^{2}\right), \\
A_{2} & =f(0.884+2.07 Z), \\
D & =1.414 Z+Z^{2}+f\left(0.754+2.657 Z+2.0 Z^{2}\right) \\
& +f^{2}\left(0.348+1.243 Z+Z^{2}\right) .
\end{aligned}
$$

Here, $p_{e}, p_{i}, T_{e}$, and $T_{i}$ denote the electron and ion pressure and temperature associated with each flux surface. derivatives are with respect to the poloidal flux within a given flux surface. $Z$ is the effective plasma charge, and $f$ is the ratio of trapped to circulating particles on a flux surface.

To derive an analytic expression for the total bootstrap current, we make the following simplifying assumptions:

$$
T_{e}=T_{i}
$$

$$
p_{e}=p_{i}
$$

(SA3) Assume a large aspect ratio expansion, use the model field variation $B=B_{0}(1+r / R \cos \theta)^{-1}$ to calculate the trapped to circulating particle fraction, and retain the leading two orders in the square root of the local inverse aspect ratio to obtain ${ }^{17}$

$$
f=1.46 \sqrt{r / R}+2.40 r / R
$$

(SA4) Assume that the temperature, pressure, and total toroidal current profiles have the parabolic form $\sigma=\sigma_{0}\left(1-r^{2} / a^{2}\right)^{\alpha_{\sigma}}$, where $\sigma=T$, $p$, and $J$. 
Then

$$
\begin{aligned}
\frac{\langle\mathbf{J} \cdot \mathbf{B}\rangle}{R\langle\mathbf{B} \cdot \nabla \phi\rangle_{b s}}=\epsilon^{1 / 2} \beta_{p}\left(\frac{I_{p}}{\pi a^{2}}\right)\left[\left(\alpha_{p} Z_{p_{1}}+\alpha_{T} Z_{T 1}\right)\right. & g_{1}\left(\alpha_{p}, \alpha_{J}\right) \\
& \left.+\epsilon^{1 / 2}\left(\alpha_{p} Z_{p_{2}}+\alpha_{T} Z_{T_{2}}\right) g_{2}\left(\alpha_{p}, \alpha_{J}\right)\right]
\end{aligned}
$$

where

$$
\begin{gathered}
g_{1}=\frac{1}{4}\left(\alpha_{p}+1\right) x^{5 / 4}(1-x)^{\alpha_{p}-1} /\left[1-(1-x)^{\alpha_{\jmath}+1}\right], \\
g_{2}=\frac{1}{4}\left(\alpha_{p}+1\right) x^{3 / 2}(1-x)^{\alpha_{p}-1} /\left[1-(1-x)^{x_{j}+1}\right] . \\
x=r^{2} / a^{2},
\end{gathered}
$$

and the coefficients $Z_{p_{1}, p_{2}}$ and $Z_{T_{1}, T_{3}}$ are cumbersome algebraic functions of the effective charge. $Z$, which are accurately represented by the rational approximations:

$$
\begin{aligned}
& Z_{p_{1}} \approx \frac{13.93+7.89 Z}{5.55 Z-1}, \\
& Z_{T_{1}} \approx-\frac{34.37+0.63 Z}{8.11 Z-1}, \\
& Z_{p_{2}} \approx-\frac{5.11-1.17 Z}{2.21 Z-1} \\
& Z_{T_{2}} \approx \frac{12.51-0.34 Z}{2.68 Z-1}
\end{aligned}
$$

Defining the total bootstrap current by

$$
I_{b s}=2 \pi \int_{0}^{a} \frac{\langle\mathbf{J} \cdot \mathbf{B}\rangle_{b s}}{R\langle\mathbf{B} \cdot \nabla \phi\rangle} r d r
$$

leads to the desired expression for the total bootstrap fraction.

$$
I_{b s} / I_{p}=\epsilon^{1 / 2} \beta_{p} C_{t s}
$$


Here

$$
\begin{gathered}
C_{b s}\left(\alpha_{n}, \alpha_{T}, \alpha_{J}, Z, \epsilon\right)=\left[\left(\alpha_{p} Z_{p_{1}}+\alpha_{T} Z_{T_{1}}\right) G_{1}+\epsilon^{1 / 2}\left(\alpha_{p} Z_{p_{2}}+\alpha_{T} Z_{T_{2}}\right) G_{2}\right], \\
G_{1}=\frac{1}{4}\left(\alpha_{p}+1\right) \int_{0}^{1} d x x^{5 / 4}(1-x)^{\alpha_{p}-1} /\left[1-(1-x)^{\alpha_{J}+1}\right], \\
C_{I_{2}}=\frac{1}{4}\left(\alpha_{p}+1\right) \int_{0}^{1} d x x^{3 / 2}(1-x)^{\alpha_{p}-1} /\left[1-(1-x)^{\alpha_{J}+1}\right],
\end{gathered}
$$

and

$$
\begin{aligned}
\beta_{p} & =\frac{3 \pi^{2}\langle p\rangle a^{2}}{\mu_{0} I_{p}^{2}} \frac{1+\kappa^{2}}{2} \\
& =\frac{4 \pi^{2} B^{2} a^{2} B}{\mu_{0}^{2} I_{p}^{2}} \frac{1+\kappa^{2}}{2}
\end{aligned}
$$

The strategy which leads from Eq. (1) to Eq. (7) involves specifying the total equilibrium current profile. The motivation behind this strategy is that MHD stability should determine the choice of desirable current profile. Once $J(r)$ is specified, the coordinate transformation $d \psi / d r=(1 / r) \int r J(r) d r$ which converts flux derivatives to radial derivatives in Eq. (1) is easily determined. The result, expressed by Eq. ( 7$)$, is a linear relation betiveen the bootstrap current, $I_{b s}$, and poloidal beta. The difference,

$$
J_{\text {seed }}(r)=J(r)-J_{b s}(r),
$$

between the specified equilibrium current density and the calculated bootstrap current density defines a seed current which must be provided by some external current drive scherne. Depending on the shape of $J_{b s}(r)$, the external current drive requirements may or may not be severe. The minimization of these requirements is discussed further in Sec. III.

A different strategy for calculating the bootstrap current has been considered by Iacono and Bhattacharjee. ${ }^{12}$ Rather than assuming a form for the equilibrium current profile, these authors assume that the external current drive scheme provides a constraint on the shape of the seed current profile. 
$J_{\text {seed }}(r)$. Consistent with this constraint, the flux coordinate transformation is written as $d \psi / d r=(1 / r) \int r\left(J_{\text {seed }}+J_{b s}\right) d r$. As a result, the dependence of $\beta_{p}$ on $I_{b s}$ is quadratic, rather than linear.

The lowest order (ie., $\sqrt{\epsilon}$ ) terms in the expression Eq. ( 7 ) for $I_{b s} / I_{p}$ were obtained previously by DeVoto et al.. ${ }^{8}$ who presented tabulated values of the $C_{1}$ integral for selected values of $\alpha_{p}$ and $\alpha_{J}$. The reason for including the order $\epsilon$ correction in the present calculation is a cancellation between $Z_{p_{1}}$ and $Z_{T_{1}}$ which increases the relative importance of the order $\epsilon$ terms when the density profile is flat so that $\alpha_{p} \approx \alpha_{T}$. For example, when the effective charge has the value $Z=2.0$, we obtain $Z_{p_{1}}=+2.94, Z_{T_{1}}=-2.34: Z_{p_{2}}=-0.81$, $Z_{T_{2}}=+2.71$. For the density, temperature, and current profile parameters $\alpha_{n}=0.5 . \alpha_{T}=1.0$. and $\alpha_{J}=1.0$. and for inverse aspect ratio $\epsilon=0.22$. we find $C_{b s}=0.54$. Of this total, $22 \%$ is the order $\epsilon$ correction. On the other hand. for $\alpha_{n}=0.1 . \alpha_{T}=1.0$, and $\alpha_{J}=1.0 . C_{b s}=0.42$ out of which $45 \%$ is the order $\epsilon$ correction.

Figure 1 shows contour plots of the bootstrap coefficient $C_{b s}$ as a function of $\alpha_{n}$ and $\alpha_{T}$ for various values of the current profile peakedness parameter, $\alpha_{J}$. Here, the inverse aspect ratio and the plasma effective charge were fixed at the values $\epsilon=0.22$ and $Z=2.0$. respectively. For the assumed parabolic current profile, an exact expression can be obtained which relates $\alpha_{J}$ to the plasma internal inductance, $\ell_{i}$. Thus,

$$
\begin{aligned}
\ell_{i} & =\frac{2}{a^{2} B_{p}^{2}(a)} \int_{0}^{a} r d r B_{p}^{2} \\
& =2 \Psi\left(\alpha_{J}+2\right)-\Psi\left(2 \alpha_{J}+3\right)-\Psi(1),
\end{aligned}
$$

where $\Psi(x)$ is a Di-gamma function. ${ }^{18}$ To avoid the necessity of a Table look-up, we may Taylor expand the $\Psi$ iunctions about $\alpha_{J}=0$ and obtain the following Pade approximation to the internal inductance of the current profile:

$$
\ell_{i} \approx \frac{1}{2}\left[\frac{1+1.1918 \alpha_{J}}{1+0.1918 \alpha_{J}}\right] .
$$

For the values of $a_{J}$ used in the Figures, evaluation of $\ell_{i}$ gives

\begin{tabular}{|l|llll|}
\hline$\alpha_{j}$ & 0 & 1 & 2 & 3 \\
\hline$\ell_{i}$ & 0.50 & 0.92 & 1.22 & 1.45 \\
\hline
\end{tabular}


It is also useful to relate the current profile parameter $\alpha_{J}$ to the shear parameter $q^{*} / q_{0}$, where $q_{0}$ is the axis value of the safety factor, and $q^{*}$ is the edge safety factor with definition

$$
q^{*}=\frac{2 \pi a^{2} B}{\mu_{0} R I_{p}}\left(\frac{1+\kappa^{2}}{2}\right) .
$$

Then

$$
\begin{aligned}
q^{*} / q_{0} & =J_{0} /\langle J\rangle \\
& =\alpha_{J}+1 .
\end{aligned}
$$

The dominant trends seen in Fig. 1 are (i) At constant $\alpha_{J}$ the coefficient $C_{b s}$ increases with increasing density peakedness, and (ii) At constant $\alpha_{n}$ and $\alpha_{T}, C_{b s}$ decreases with increasing current peakedness. For example, with $\alpha_{J}$ and $\alpha_{T}$ fixed at nominal values 1.0 and 1.5, respectively, $C_{b s}$ increases from 0.41 to 0.66 when $\alpha_{n}$ is increased from 0.0 to 1.0 ; and with $\alpha_{n}$ and $\alpha_{T}$ fixed at the values 0.5 and 1.5 , respectively, $C_{b s}$ decreases from 0.86 to 0.46 when $\alpha_{J}$ increases from 0.0 to 2.0 .

For contour plotting the function $I_{b s} / I_{p}$, it is convenient to fold the profile dependence of poloidal beta into the profile dependence of $C_{b s}$. To do this we express the toroidal beta as a fraction, $f$, of the Troyon beta limit:

$$
3=f 3_{\text {Troy }}
$$

where

$$
\beta_{\text {Troy }}=C_{T} \frac{\mu_{0}}{40 \pi} \frac{I_{p}}{a B}
$$

and $C_{T}$ is the Troyon coefficient. Then Eqs. (9), (14)-(17) give

$$
\beta_{p}=0.05 f C_{T}^{\prime} q_{0} \epsilon^{-1}\left(\alpha_{J}+1\right),
$$

and the bootstrap fraction, Eq. (7), becomes

$$
\dot{I}_{b s} / I_{F}=\frac{0.05 f C_{T q_{0}}}{\sqrt{\epsilon}}\left(\alpha_{J}+1\right) C_{b s} .
$$


Figure 2 shows (solid) contours of the right hand side of $\mathrm{Eq}$. (19) for selected values of $\alpha_{J}$ and the restriction $f C_{T} q_{0}=3.5$. This corresponds to the evaluation of the bootstrap fraction at a nominal beta limit $f=1$, with $q_{0}=1$ and a Troyon coefficient $C_{T}=3.5$. However, since the right hand side of Eq. (19) is linear in $f q_{0}$, it follows that the value of $I_{b s} / I_{p}$ can be read from Fig. 2 for any $\beta$ or $q_{0}$ by application of a linear scaling. A discussion of the results shown in Fig. 2 is given in the next Section.

\section{Bootstrap Current Alignment}

To realize the potential benefits of operating with a bootstrap fraction $I_{b s} / I_{p}$ near unity, the bootstrap profile must have an appropriate shape. For example, suppose we want to produce a (total) current density profile with a predetermined shape, such as shown in Fig. 3. If the dominant current drive is to be bootstrap, it would clearly be undesirable to generate a bootstrap current density, $J_{b s}$, which peaks near the plasma edge, as in Fig. 3(b). If this were so, some other means of current drive (such as lower hybrid, etc.,) would not only have to supply the current deficit at the plasma center to obtain the desired total profile, but also would have to provide reverse drive near the edge. A more desirable shape for the bootstrap current density is shown in Fig. 3(a), where the peak in $J_{b s}$ is seen to occur near the plasma center. In this case. the additional current drive needs to be driven near the center, and is predominantly unidirectional. We would like to determine which regions of parameter space correspond to "desirable" bootstrap profiles in the sense of Fig. 3(a).

For the parabolic profiles of density, temperature, and current density assumed in this paper the bootstrap current density is constrained to vanish at the origin $(r=0)$, the plasma edge $(r=a)$, and has a single maximum at some intermediate radial location. This implies that once the magnitude of the bootstrap current is specified, the shape of $J_{b s}$ can be characterized by a single parameter, namely the location of the turning point, $x_{t}$, such that $d J_{b s}\left(x_{t}\right) / d x=0$. If the total bootstrap fraction is close to unity, we may identify smail $x_{t}$ as being desirable and large $x_{t}$ as undesirable.

It is convenient to derive the dependence $x_{t}\left(\alpha_{n}, \alpha_{T}\right)$ on the profile parameters $\alpha_{n}$ and $\alpha_{T}$ for a given $\alpha_{J}$ so that contours of $x_{t}$ can be superimposed on the plots of $I_{b s} / I_{p}$, thereby providing information on a single plot of both 
shape and magnitude of the bootstrap current. The condition for $x_{t}$, ie., that the derivative of the right hand side of Eq. (3) vanishes, can be easily derived. Inspection of Eq. (3) shows that if the order $\epsilon$ terms are neglected and $\alpha_{J}$ is fixed, then lines of constant $x_{t}$ are parallel to lines of constant $\alpha_{p}$. (Such lines would have slope $=-1$ in Fig. 1 and Fig. 2). Including the order $\epsilon$ terms skews the alignment of $x_{t}$ with $\alpha_{p}$ (because of the $\alpha_{T}$ dependence), particularly at small $\alpha_{n}$ when these terms are large. However the skewness is small, as can be seen in Fig. 2, where the dashed lines are exact contours of $x_{t}\left(\alpha_{n}, \alpha_{T}\right)$.

From Fig. 2 various trelils are evident:

With regard to the magnitude of the bootstrap fraction at the beta limit.

(T1) For fixed density and temperature profile shapes, the value of $I_{h s} / I_{p}$ increases with increasing $\alpha_{J}$. This contrasts with the trend for $C_{b s}$, showing that the dependence of $C_{b s}$ on the current profile shape is weaker than $\alpha_{J}+1$. For example, with $\alpha_{n}$ and $\alpha_{T}$ fixed at the values 0.5 and 1.5, respectively, the bootstrap fraction at the nominal beta limit increases from $I_{b s} / I_{p}=0.32$ to $I_{b s} / I_{p}=0.51$ when $\alpha_{J}$ increases from 0.0 to 2.0 .

(T2) For any fixed current profile shape, the value of the bootstrap fraction, $I_{b s} / I_{p}$, increases strongly with increasing $\alpha_{n}$. The variation of $I_{b s} / I_{p}$ with $\alpha_{T}$ is extremely weak. For example, consider a reference state with $\alpha_{n}=0.5$, $\alpha_{T}=1.5$, and $\alpha_{J}=0.0$. Then increasing $\alpha_{n}$ from 0.5 to 1.0 increases $I_{b s} / I_{p}$ from 0.32 to 0.40 , while increasing $\alpha_{T}$ from 1.5 to 2.0 increases $I_{h s} / I_{p}$ from 0.32 to 0.34 . Similarly, if the reference state has a more peaked current profile with $\alpha_{n}=0.5, \alpha_{T}=1.5$, and $\alpha_{J}=2.0$, then increasing $\alpha_{n}$ from 0.5 to 1.0 increases $I_{b s} / I_{p}$ from 0.51 to 0.60 , while increasing $\alpha_{T}$ from 1.5 to 2.0 keeps $I_{b s} / I_{p}$ at the value 0.51 .

With regard to the alignment of the bootstrap current profile,

(T3) For fixed current profile shape, the bootstrap profile peaks at smaller minor radius as $\alpha_{p}$ is increased, whether by increasing $\alpha_{n}$ or $\alpha_{T}$.

(T4) For fixed $\alpha_{p}$, the bootstrap current profile peaks at smaller minor radius as $\alpha_{J}$ is decreased.

It is interesting to note that the desire to minimize the external current drive requirements for tokamak reactors implies a desire for large $I_{b s} / I_{p}$ and 
small $x_{t}$. We are led, therefore, to a need to compromise between the trends (T1) and (T4)). Also, with regard to the possibility of an essentially $100 \%$ bootstrap driven tokamak, we note that the $I_{b s} / I_{p}=1.0$ contour has not entered the plots even when $\alpha_{J}$ has been raised to 3.0. To obtain $I_{b s} / I_{p} \approx 1.0$, we need to assume $f C_{T} q_{0}>3.5$. Operating in the Second Stable region, where $f C_{T}>3.5$, and $q_{0}>1.0$, offers a possible solution, as can be seen from Fig. 2 by the linear scaling of the contour levels of $I_{b s} / I_{p}$ mentioned at the end of Sec. 2.

\section{Comparison of Results with a Fully Toroidal Equi- librium Calculation}

In order to test how well the formulas Eq. ( 7 ) and Eq. (19) can reproduce the output from a fully toroidal MHD equilibrium code, we present a comparison of results in Table 1 and Figs. 4 and 5. Four MHD equilibria corresponding to different choices for the input profiles $J(\psi), p(\psi)$ and $T(\psi)$ were computed using the Princeton JSOLVER equilibrium code. Each input profile is a two-parameter function of the poloidal flux, $\psi$, of the form

$$
\sigma^{J S}(\psi)=\sigma(0)\left(1-\psi^{b}\right)^{a},
$$

with $\sigma=J, p$, and $T$. The variation of $b$, in addition to $a$, allows vailoring of the profile shapes. For the calculations, the major radius, minor radius, elongation, and triangularity of the outermost flux surface were fixed at the values $R=2.25 \mathrm{~m}, a=0.5 \mathrm{~m}$, (i.e., $\epsilon=0.22$ ), $\kappa=2.0$, and $\delta=0.5$. The toroidal magnetic field was $B_{t}=3.3 \mathrm{~T}$. Once an equilibrium is calculated, the bootstrap current density on each flux surface is evaluated using Eq. (1.) with $T_{e}=T_{i}, p_{e}=p_{i}$, and $Z=2.0$. The current density is then integrated over the flux to yield the total bootstrap current, $I_{b s}$.

To evaluate the approximation given by Eq. (7) for the bootstrap fraction, values for the profile exponents $\alpha_{\sigma}(\sigma=p, T$, and $J)$ which provide the oneparameter description of the model profiles are needed. For this purpose, the identification

$$
\alpha_{\sigma}=\sigma(0) /\langle\sigma(\psi)\rangle-1
$$

is made, where the peak-to-average ratios on the right hand side are taken from the output of the equilibrium runs. In a cylinder. Eq. (21) is exact for 
parabolic profiles of the form (SA4). The peak-to-average ratios, together with the poloidal beta, Troyon coefficient, central and edge safety factors, and total plasma current. are presented in Table 1. The value of $C_{b s}$ given by Eq. (8) is also presented, using the prescription in Eq. (21). The final two columns of the Table show the bootstrap current fraction calculated by the equilibrium code, and the fraction obtained by evaluation of Eq. ( 7 ). In spite of the considerable variation in profile parameters, excellent agreement between the JSOLVER and model expressions for $I_{b s} / I_{p}$ is found in all cases.

Figure 4 shows JSOLVER equilibrium plots of the equilibrium and bootstrap current profiles $J(\psi) / J(0)$, and $J_{b s}(\psi) / J_{b s}(0)$, respectively. The poloidal flux is normalized in the pots to equal unity at the plasma edge and zero at the magnetic axis. For comparison. Fig. $j$ shows plots of the model profiles $J(x) / J_{0}=(1-x)^{\text {crs }}$ and $J_{b s}(x) / J_{b s}(0)$ obtained from Eq. (3). The chosen abscissa is $x=r^{2} / a^{2}$, so that, as in Fig. 4 , the area under each curve is the total current. In addition to the agreement between the integrated bootstrap current profiles evidenced in Table 1, the model bootstrap profiles shown in Figs. 5(a), (c), and (d) show substantial agreement in shape with the MHD equilibrium profiles (see Figs 4(a), (c), and (d)). However, when comparing Figs. 4 and 5 we should be aware of the missing Jacobian factor $\partial \psi / \partial(x)$ which relates the absissae, and that the one-parameter description of the model plasma profiles using Eq. (21) can sometimes lead to significant detailed differences. Thus, for example, the solid curves in Figs. 4(a) and $5(\mathrm{a})$ show a different radial dependence of the equilibrium and model $J$ profile shapes, particularly near the plasma center. More apparent is the difference between the bootstrap current profiles shown in Figs. $4(\mathrm{~b})$ and $5(\mathrm{~b})$, where the equilibrium code evaluation of $J_{b s}(\psi)$ shows a much smaller value over a substantial region near the origin than does the model calculation. In this case, the difference can be traced to the fact that the two-parameter deccription of the pressure profile for the exact equilibrium calculation is poorly described by the single moment described by Eq. (21): The pressure gradient for the equilibrium calculation near the magnetic axis is considerably weaker than the single moment description predicts, leading to a substantially smaller local bootstrap current drive.

To illustrate the use of Figs. 1 and 2 for modifying operating scenarios, we note that although the range of bootstrap current fractions appearing in Table 1 spans a wide range (from approximately 0.4 to 1.0 ), the variation is 
almost entirely due to differences in $\beta_{p}$. In fact, the bootstrap coefficient $C_{b s}$ only varies between 0.43 and 0.47 for the four equilibrium scenarios. Now suppose that scenario (d) is perceived to be too aggressive from the roint of view of 3 , yet the value of $I_{h s} / I_{p}$ is required to be held fixed at approximately unity. Then we note from Fig. 1 that increasing the density peaking factor from $\alpha_{n}=0.06$ to $\alpha_{n}=0.5$ has the effect of increasing $C_{b s}$ by $20 \%$. Thus, 3 may be lowered by $20 \%$ at no expense to the bootstrap fraction if this modification to the density profile can be realized.

\section{Summary}

Contour plots of the bootctrap coefficient $\left(c_{s s}=\left(I_{b s} / I_{p}\right) / e^{1 / 2}, 3_{p}\right.$. and of the bootstrap current fraction $I_{h s} / I_{p}$ evaluated at the first stability beta limit. have been presented in Figs. 1 and 2 as a function of density and temperature peaking factors $\alpha_{n}$ and $\alpha_{T}$. These comprehensive plots are shown for fixed effective charge. Z. and inverse aspect ratio. $\epsilon$. and various currert profile peaking factors. $a_{J}$. The trends displayed in the figures can be used 10 identify possible operating regimes for tokamak reactors which make efficient use of large bootstrap fractions.

Major trends show that the values of $C_{b s}$ and of $I_{b s} / I_{p}$ at the beta limit increase strongly with increasing $\alpha_{n}$ This clearly shows the benefit of being able to peak the density profile. Also. the location of the maximum of the bootstrap current density moves to smaller minor radius when the pressure profile is peaked, either by increasing $\alpha_{n}$ or by increasing $\alpha_{T}$. Causing the 'notstrap current to peak ai small radius is important if the bootstrap current fraction is desired to be near unity. since otherwise a substantial bootstrap overdrive in the vicinity of the bootstrap maximum will result. and the requirements of an external current drive systern which must in any event provide a plasma seed near the axis will be increased.

Finally. while the value of $C_{b s}$ decreases with increasing $\alpha_{J}$, the value of $I_{b s} / I_{p}$. at any fixerl volume averaged beta, increcises with increasing current peakedness. This desirable effect. however. competes with a trend for the location of the bootstrap maximum to move to larger minor racius when $\alpha_{J}$ is increased.

Despite the clear trends which emerge from the zero-dimensional analysis which gave rise to the Figs. 1 and 2. it is important to note that the attempt 
to use the figures to optimize the use of large bootstrap current fractions has neglected the important issue of MHD stability. However, the above analysis should provide a guideline for a more thorough two-dimensional equilibrium and stability treatment. 
Table 1: Profile Parameters and Bootstrap Fractions for Four Equilibria.

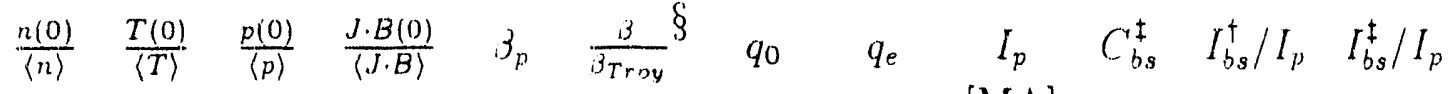

$$
\begin{aligned}
& \text { [MA] } \\
& \begin{array}{llllllllllll}
1.86 & 3.82 & 4.83 & 3.98 & 0.64 & 0.64 & 0.71 & 10.79 & 1.21 & 0.44 & 0.42 & 0.40
\end{array} \\
& \begin{array}{llllllllllll}
1.41 & 2.08 & 2.37 & 2.43 & 0.65 & 0.94 & 1.02 & 5.95 & 1.50 & 0.48 & 0.53 & 0.52
\end{array} \\
& \begin{array}{llllllllllll}
1.49 & 2.57 & 3.14 & 3.41 & 0.89 & 0.85 & 1.02 & 11.59 & 1.00 & 0.43 & 0.69 & 0.63
\end{array} \\
& \begin{array}{llllllllllll}
1.06 & 3.65 & 3.72 & 2.09 & 1.33 & 1.13 & 2.01 & 10.67 & 0.89 & 0.47 & 0.96 & 1.02
\end{array} \\
& \S \text { Assumes } C_{T}=3.5 \mathrm{in} \mathrm{Eq.} \mathrm{(1i).} \\
& \dagger \text { JSOLVER output. } \\
& \ddagger \text { From Eq. (7). }
\end{aligned}
$$

Acknowledgments It is a pleasure to acknowledge useful discussions with D. Ehst. R. Iacono, S. C. Jardin, C. Kessel, W. Nevins, R. Perkins, and P. Rutherford. I would particularly like to thank C. Kessel for providing invaluable help with the JSOLVER equilibriun calculations.

This work was supported by US Department of Energy contract number DE-AC02-76-CHO3073. 


\section{References}

'R. J. Bickerton. J. W. Connor, and J. B. Taylor, Nature 229. 110, (1971)

2Y. C. Zarnstorff, Phys. Rev. Lett. 60, 1306, (1988)

${ }^{3}$ J. G. Cordey, C. D. Challis, and P. M. Stubberfield, Plasma Phys. 30, 1625. (1988)

${ }^{4}$ G. Becker Nucl. Fusion 29, 1291, (1989)

${ }^{5}$ M. Kikuchi. M. Azumi, K. Tani, and H. Kubo, Nucl. Fusion 30, 343, (1990)

${ }^{\circ}$ F. Najmabadi. R. W. Conn, é, al., "The ARIES-1 Tokamak Reactor Study, Final Report. 1991": ICLA-PPG-1323

D. A. Ehst. and K. Evans, Argonne National Laboratory Report ANL/FPP/TM-235 (1989)

${ }^{8}$ R. S. DeVoto. M. E. Fenstermaker and A. A. Mirin, Nucl. Fusion 29, 1769, (1989)

${ }^{9}$ V. D. Pustovitov, JETP Lett., 54, 313, (1991)

${ }^{10}$ H. R. Wilson Nuci. Fusion 32, 257, (1992)

${ }^{11}$ G. R. Harris "Comparision of Different Bootstrap Current Expressions" EUR-CEA-FC-1436. November 1991

${ }^{12}$ R. Iacono, and A. Bhattacharjee Phys. Fluids B4,1685, (1992)

${ }^{13} \mathrm{R}$. H. Weening 'Beta limit of a Completely Bootstrapped Tokamak", CRPP Lausanne LRP 453/92 . Warch 1992.

${ }^{14} \mathrm{~F}$. W. Perkins 'Scaling Considerations for High Bootstrap Fraction Tokamaks", (subrnitted for publication in Nucl. Fusion 1992)

${ }^{15}$ A. H. Boozer Phys. Fluids 29, 4123. (1986)

${ }^{16}$ R. H. Weening and A. H. Boozer Phys. Fluids B4, 159. (1992)

${ }^{17}$ S. P. Hirshman. Phys. Fluids 31. 3150, (1988) 
${ }^{18} \mathrm{M}$. Abramowitz and I. Stegun, Editors, Handbook of Mathematical Functions, National Bureau of Standards, Washington D.C., 1964 


\section{Figures}

FIG. 1. Contour plots of the bootstrap coefficient $C_{b s}(\mathrm{Eq} .(T))$ for various values of $\alpha_{J}$, and fixed $\epsilon=0.22, Z=2.0$.

FIG. 2. Contour plots of the bootstrap fraction $I_{b s} / I_{p}$ evaluated at the beta limit as a function of $\alpha_{n}$ and $\alpha_{T}$ for various values of $\alpha_{J}$, and fixed $\epsilon=0.22$, $Z=2.0$.

FIG. 3. Schematic picture of "desirable" and "undesirable" bootstrap current profiles $J_{b s}$.

FIG. 4. Plots of the total equilibrium current density (solid), and bootstrap current density (dashed) calculated using the JSOLVER MHD equilibrium code. Abscissa is poloidal flux.

FIG. 5. Plots of the total current density, $J / J_{0}=\left(1-r^{2} / a^{2}\right)^{\alpha}$, and approximate bootstrap current density, $J_{b s} / J_{0}$ from Eq. (3). 

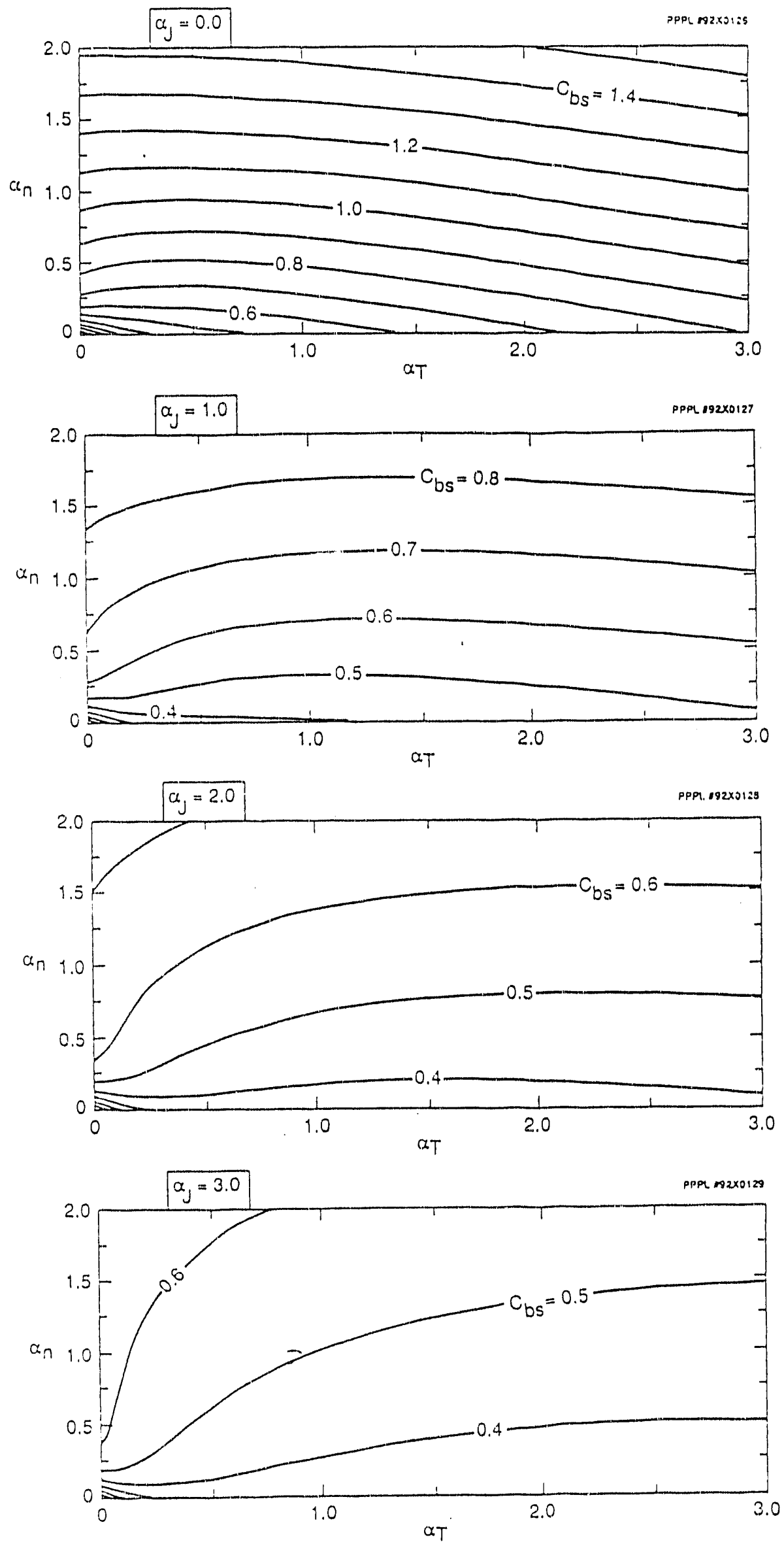

Figure 1 

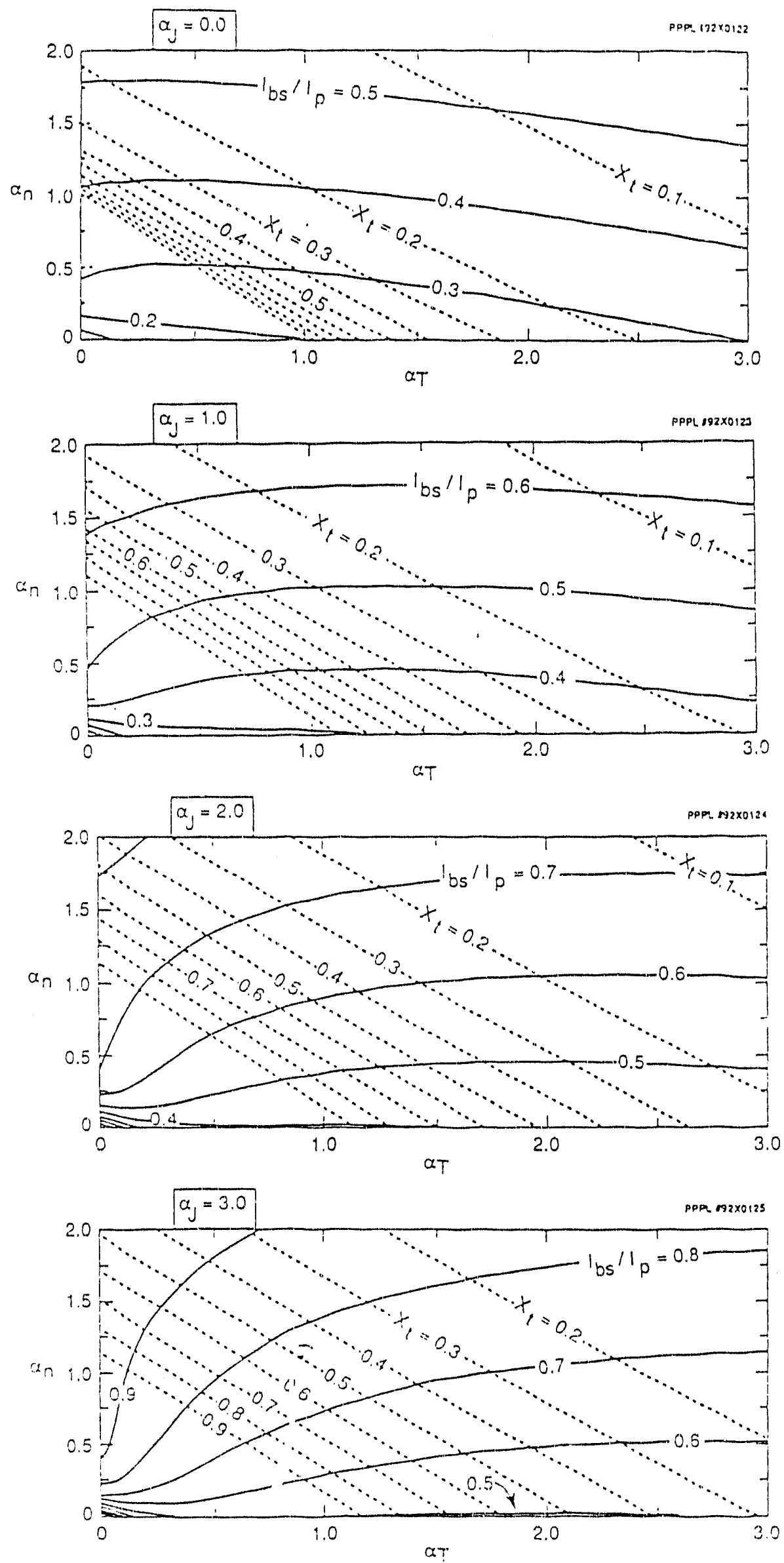

Figure 2 

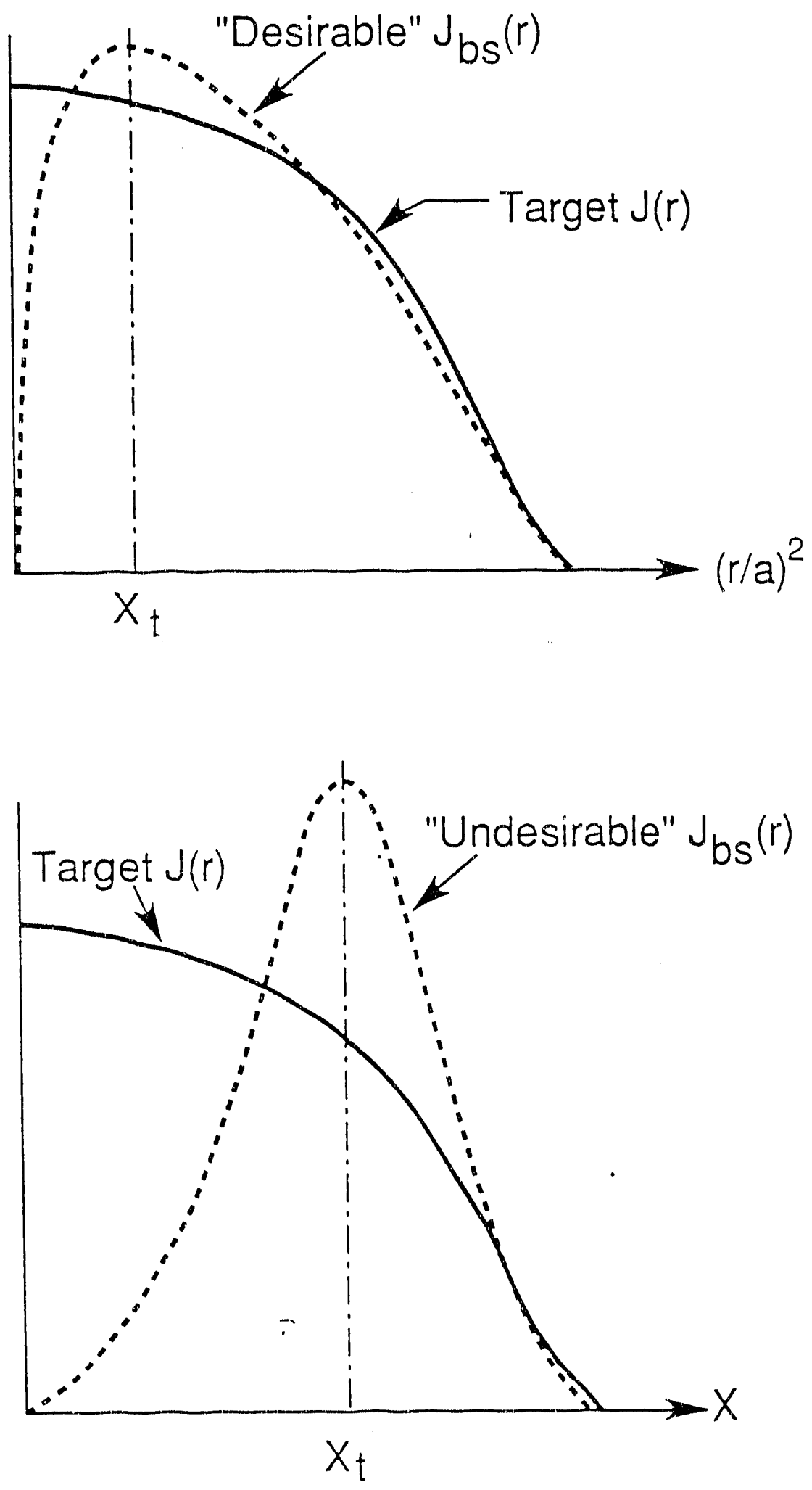

Figure 3 

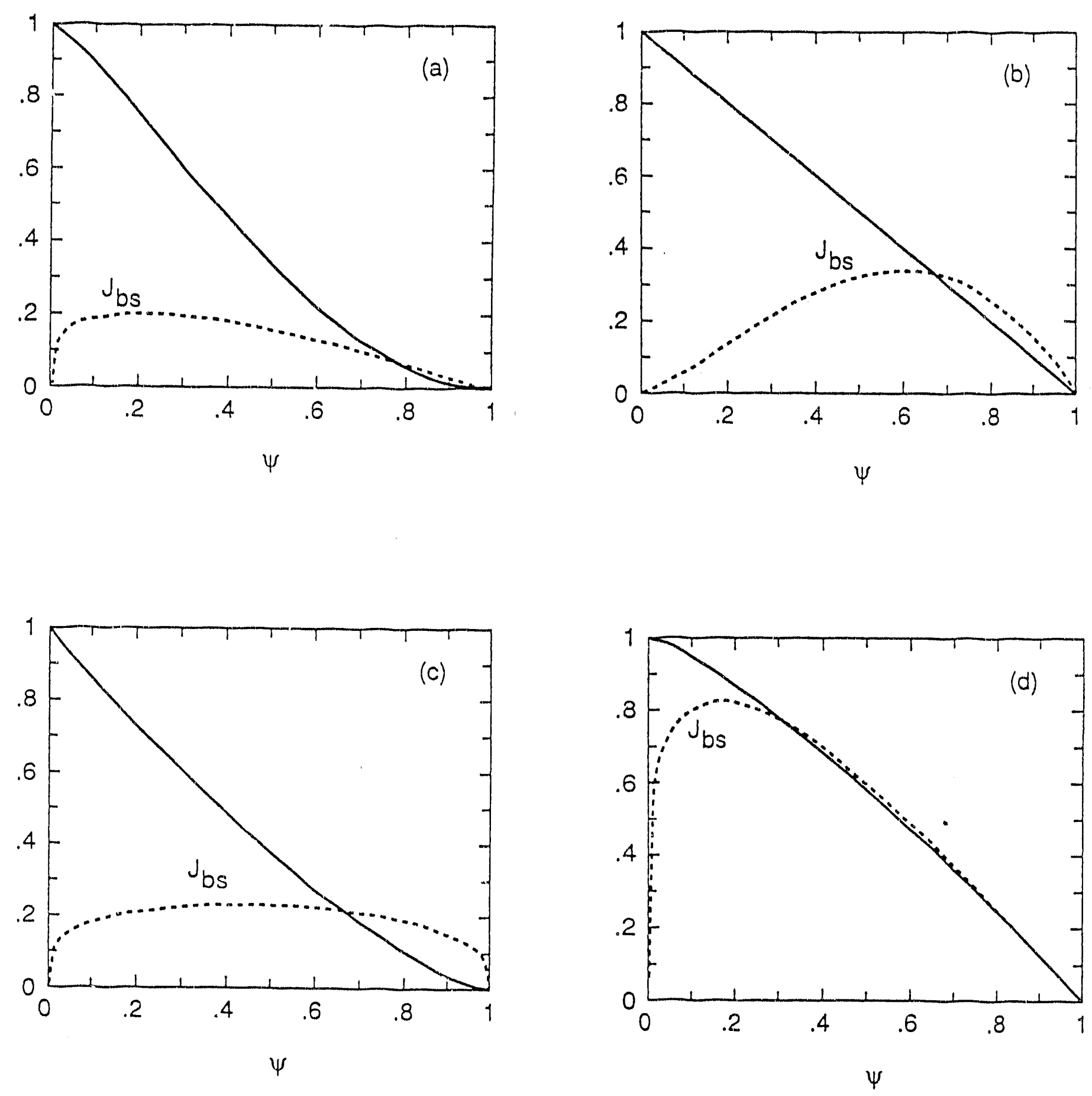

Figure 4 

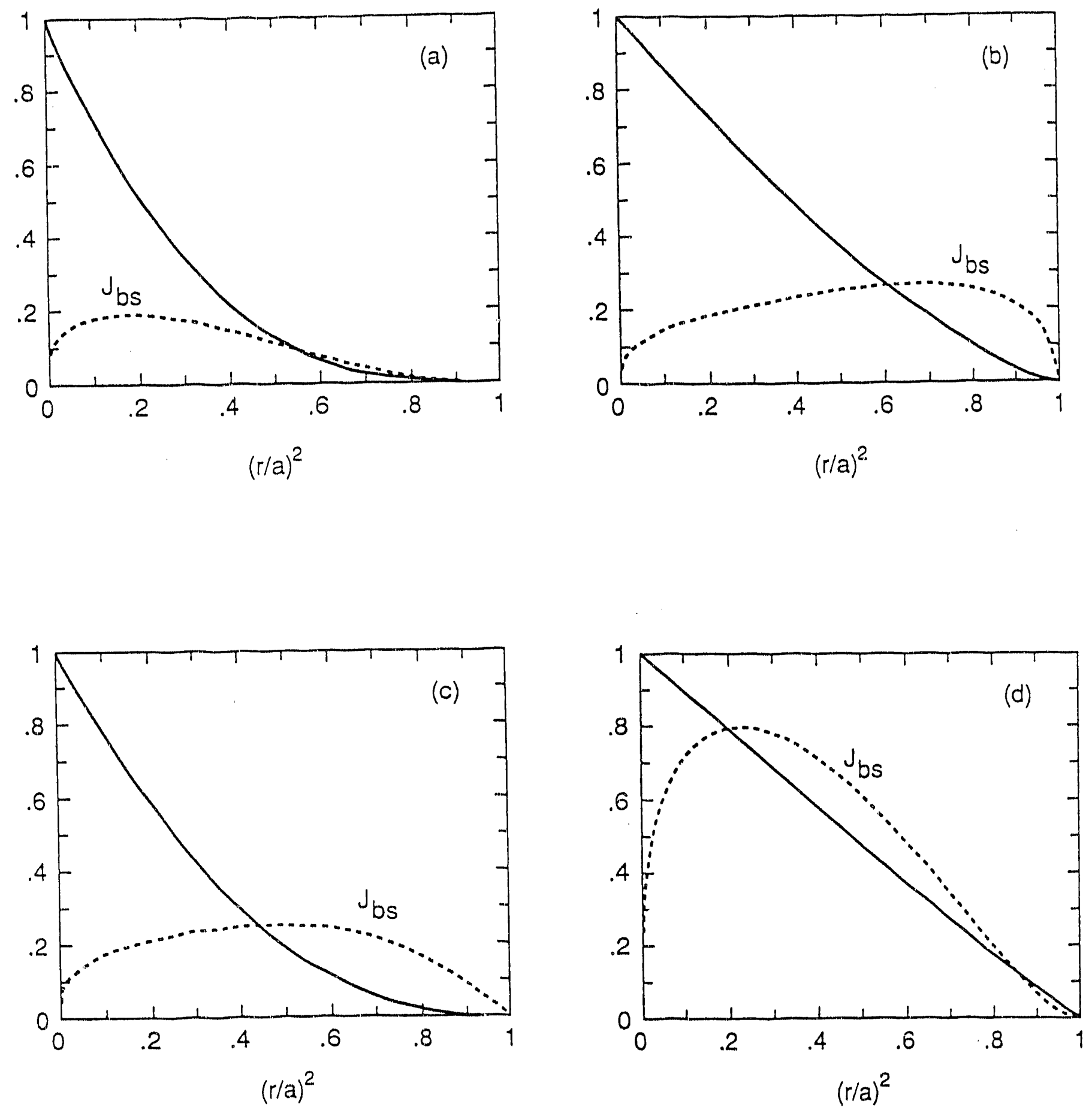

Figure 5 
Dr. F. Proboni, Univ, of K'ollongong, AUSTRALIA

Prot. M.K. Brennan, Univ. of Syonoy, AUSTRALIA

Plasma Rosearch Lob., Aurstation Nat. Univ., AUSTRALIA

Prof. I.R. Jones, Finders Univ, AUSTRALIA

Prot. F. Cap, Inst for Theoreticel Physics, AUSTRIA

Prot. M. Heinciler, Institut for Theoretische Physik, AUSTRIA

Prof. M. Gooseems, Astronomicch InstituUt, BELGIUM

Ecolw Roydo Milituro, be do Pry. Plaemas, BELGIUM

Cormmiscion-Europmen, DQ. XII-FUsion Prog., BELGIUM

Prot. R. Bouctaus, Nixkunimeribitit Gent, BELGIUM

Dr. P.H. Sakencik, Instiuto Fisica, BRLZIL.

Instituto Neoiond Do Posquisas Espaciaie-INPE, BRZIL

Dooumante Orinos, Abornic Energy of Cenede Ldo., CANADA

Dr. M.P. Bectynekj, MPB Technologies, Inc., CANADA

Dr. H.M. Skaragard, Univ. of Suckatchewen, CANADA

Prof. J. Toichmann, Univ. of Aontreal, CANADA

Prof. S.R. Sreenivasen, Uniy. of Coly ary, CANADA

Prot. T.W. Jometon, INAS-Energio, CANADA

Dr. R. Botton, Centre canadion de tusion megnetique, CANADA

Dr. C.R James, Univ. of Nborta. CANAOA

Dr. P. LukAc, Komensktho Universzita, CZECHOSLOVAKIA

The Librarian, Cutham Letooratory. ENGLAND

Library, AS1, Ruthertord Applotion Laboratory, ENGLAND

Wirs. S.A. Hutchinson, JET Librery. ENGLAND

Dr. S.C. Shame, Univ. of South Pacific, FIJI ISLANDS

P. Matonen, Univ. of Hedsinki, FINLAND

Prof. M.N. Busesec, Ecots Potyrachnique., FRANCE

C. Moumat, Lob, do Physique des Nilioux bnisos, FRANCE

J. Racht, CENCADARACHE - Bat 506, FPANCE

Prof. E. Economow, Univ. of Cron, GREECE

M6. C. Rinni, Univ, of loamina, GREECE

Dr. T. Mud, Acactemy Bibliographic Ser., HONG KONG

Preprint Library, Hungarian Acadamy of Sai., HUNGARY

Dr. B. DesGupta, Saha inst of Nucteer Prysics, INDIA

Dr. P. Kaw, Inst. for Piasma Rosearch, INDIA

Dr. P. Rosensu, Iereed inse of Technology, ISRAEL

Librenian, Intomational Conter for Theo Physics, ITALY

Miss C. Do Palo, Associazion EURATONANEA, ITALY

Dr. G. Grosso, Istituto di Fieica del Plaema, ITALY

Prof. G. Rostangni, Istwio Gas lonizzati Del Cnr, ITALY

Dr. H. Yemeto, Toshibe Ros Devel Conter, JAPAN
Prof. I. Kawakami, Hiroshima Univ., JAPAN

Prof. K. Nishikawa, Hirostima Univ., JAPAN

Director, Jepen Atomic Enorgy Research inst., JAPAN

Prot. S. Itoh, Kyuehu Univ., JAPAN

Researn Into. Ct., Nationa Instit for Fusion Science, JAPAN

Prot. S. Tanake, Kyoto Univ., JAPAN

Library, Kyoso Univ., JAPAN

Prot. N. inow. Univ. of Tokyo, LAPAN

Secresery, Pleama Section, Elactrotechnical Lab., JAPAN

S. Mori, Techniced Atriser, LAERI, LAPAN

D. O. Mided, Kunanets Inat of Technotooy, JAPAN

J. Hyeon-Sook, Korea Atomic Enorgy Posearch Inst. KOREA

D.I. Chal, The Kon Adv. Inst. of Sai. \& Toch., KOREA

Prot. B.S. Liby, Univ. of Waikato, NEW ZEALAND

Inst of Phyeica, Chinowe Acad Sci PEOPLE'S REP. OF CHINA

Librey, Inst of Plasma Physics, PEOPLE'S REP. OF CHINA

Teinghua Univ. Librory, PEOPLE'S REPUBLIC CF CHINA

2. Li, S.W. Ina Physics, PEOPLE'S REPUBUC OF CHINA

Prof. J.A.C. Cabrd, Instituto Superior Tecnico, PORTUGAL

Dr. O. Ponus, Al I Cuza Univ., Romania

Dr. J. Vuliars, Fusion Studios, AEC, S. AFRICA

Prof. M.A. Hetorg, Univ. of Nadid, S. AFAICA

Prot. D.E. Kim. Poheng Inat. of Sc. \& Tech., SO. KOAEA

Prof. C.I.E.M.A.T, Fusion Division Library, SPAIN

Dr. L Stomio, Univ. of UMEA, SWEDEN

Librery, Royd Inat. of Tectnology, SWEDEN

Prof. H. Whatmeon, Chalmers Univ. of Tech., SWEDEN

Contre Phys. Dos Pleemas, Ecolo Polytech, SWITZERLAND

Bibliothen, Inat. Voor Plasma-Fyaica, THE NETHERLANDS

Asst Prot. Dr. S. Cakir, Eidd East Tech. Univ., TURKEY

Dr. V.A. Gubtikh, Sai. Res. Inst. Electrophys.I Apparatus, USSR

Dr. D.D. Ayutov, Siberian Brench of Acadomy of Sod., USSA

Dr. G.A. Eliser, I.V. Kurchato Inst, USSA

Librarien, The Ukr.SSA Acedemy of Scioncos, USSA

D. LM. Kovrizhnykh, Inst. of Gervera Physics, USSR

Kentorschungendoge GmbH, Zentrabibliothok, W. GEAMANY

Bibliothok, Inst FOr Plasmatorechung, W. GEAMANY

Prot. K. Schindor, Ruthr-Uninersith Bochum, W. GERMANY

Dr. F. Wogner, (ASOEX), Max-Plenck-Inetitut, W. GERMANY

Litrerien, Max-Plenct-Inetitut, W. GERMANY

Prof. R.K. Janew, Inat of Prysics, YUGOSLAVIA 

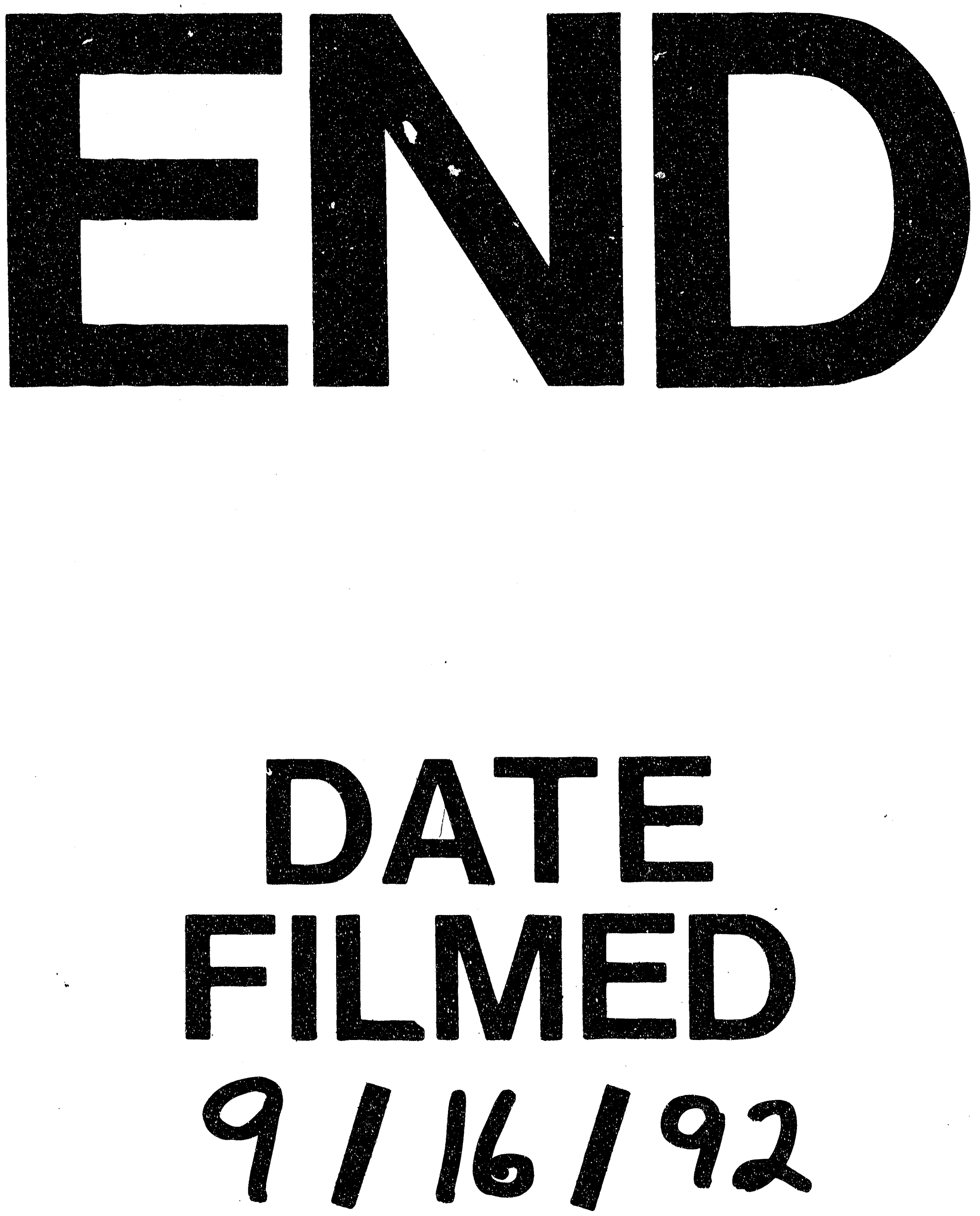
